\title{
Randomized trial of community health worker-led decision coaching to promote shared decision-making for prostate cancer screening among Black male patients and their providers
}

Danil V. Makarov ${ }^{1,2,3^{*}}$ (D), Zachary Feuer ${ }^{1,2}$, Shannon Ciprut ${ }^{1,2,3}$, Natalia Martinez Lopez ${ }^{3}$, Angela Fagerlin ${ }^{4}$, Michele Shedlin ${ }^{5}$, Heather T. Gold ${ }^{3}$, Huilin Li ${ }^{3}$, Gina Lynch ${ }^{6}$, Rueben Warren${ }^{7}$, Peter Ubel ${ }^{8}$ and Joseph E. Ravenell ${ }^{3}$

\begin{abstract}
Background: Black men are disproportionately affected by prostate cancer, the most common non-cutaneous malignancy among men in the USA. The United States Preventive Services Task Force (USPSTF) encourages prostate-specific antigen (PSA) testing decisions to be based on shared decision-making (SDM) clinician professional judgment, and patient preferences. However, evidence suggests that SDM is underutilized in clinical practice, especially among the most vulnerable patients. The purpose of this study is to evaluate the efficacy of a community health worker (CHW)-led decision-coaching program to facilitate SDM for prostate cancer screening among Black men in the primary care setting, with the ultimate aim of improving/optimizing decision quality.

Methods: We proposed a CHW-led decision-coaching program to facilitate SDM for prostate cancer screening discussions in Black men at a primary care FQHC. This study enrolled Black men who were patients at the participating clinical site and up to 15 providers who cared for them. We estimated to recruit 228 participants, ages 40-69 to be randomized to either (1) a decision aid along with decision coaching on PSA screening from a CHW or (2) receiving a decision aid along with CHW-led interaction on modifying dietary and lifestyle to serve as an attention control. The independent randomization process was implemented within each provider and we controlled for age by dividing patients into two strata: 40-54 years and 55-69 years. This sample size sufficiently powered the detection differences in the primary study outcomes: knowledge, indicative of decision quality, and differences in PSA screening rates.

Primary outcome measures for patients will be decision quality and decision regarding whether to undergo PSA screening. Primary outcome measures for providers will be acceptability and feasibility of the intervention. We will examine how decision coaching about prostate cancer screening impact patient-provider communication. These (Continued on next page)
\end{abstract}

\footnotetext{
* Correspondence: Danil.Makarov@nyulangone.org

'VA New York Harbor Healthcare System, 423 E 23rd St, New York, NY, USA

${ }^{2}$ Departments of Urology, NYU Langone Health, 227 E 30th St, New York, NY,

USA

Full list of author information is available at the end of the article
}

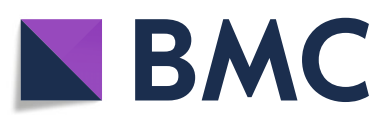

(- The Author(s). 2021 Open Access This article is licensed under a Creative Commons Attribution 4.0 International License, which permits use, sharing, adaptation, distribution and reproduction in any medium or format, as long as you give appropriate credit to the original author(s) and the source, provide a link to the Creative Commons licence, and indicate if changes were made. The images or other third party material in this article are included in the article's Creative Commons licence, unless indicated otherwise in a credit line to the material. If material is not included in the article's Creative Commons licence and your intended use is not permitted by statutory regulation or exceeds the permitted use, you will need to obtain permission directly from the copyright holder. To view a copy of this licence, visit http://creativecommons.org/licenses/by/4.0/ The Creative Commons Public Domain Dedication waiver (http://creativecommons.org/publicdomain/zero/1.0/) applies to the data made available in this article, unless otherwise stated in a credit line to the data. 
(Continued from previous page)

outcomes will be analyzed quantitatively through objective, validated scales and qualitatively through semistructured, in-depth interviews, and thematic analysis of clinical encounters. Through a conceptual model combining elements of the Preventative Health Care Model (PHM) and Informed Decision-Making Model, we hypothesize that the prostate cancer screening decision coaching intervention will result in a preference-congruent decision and decisional satisfaction. We also hypothesize that this intervention will improve physician satisfaction with counseling patients about prostate cancer screening.

Discussion: Decision coaching is an evidence-based approach to improve decision quality in many clinical contexts, but its efficacy is incompletely explored for PSA screening among Black men in primary care. Our proposal to evaluate a CHW-led decision-coaching program for PSA screening has high potential for scalability and public health impact. Our results will determine the efficacy, cost-effectiveness, and sustainability of a CHW intervention in a community clinic setting in order to inform subsequent widespread dissemination, a critical research area highlighted by USPSTF.

Trial registration: The trial was registered prospectively with the National Institute of Health registry (www. clinicaltrials.gov), registration number NCT03726320, on October 31, 2018.

Keywords: Prostate cancer, PSA, Racial disparity, Screening, Shared decision-making, Community health worker, Randomized controlled trial

\section{Background}

Prostate cancer is the second leading cause of cancer death among men in the US [1] and harms Black men disproportionately [2]. Prostate cancer comprises nearly one third of all new cancers in Black men, affecting almost 30,000 men each year. The incidence rate is $70 \%$ higher for Black men compared with other racial and ethnic groups, and mortality is 2-3 times higher compared with White men. Low socioeconomic status has been found to be a predictor of poor prostate cancer outcomes, including mortality; however, the BlackWhite disparity persists even when accounting for all of these other factors [3].

Prostate cancer mortality has declined since the adoption of PSA screening. While some of this benefit is a result of screening, the exact effect is difficult to quantify $[4,5]$. Although screening unquestionably detects many early-stage cancers, over-diagnosis of indolent cancers, leading to over-treatment, remains a significant concern $[6,7]$. The USPSTF recommends an informed decisionmaking, or "shared decision-making" (SDM) approach, to PSA screening between men aged 55 to 69 years and their healthcare providers, and other organizations recommend screening Black men at age 40 [8]. SDM includes three critical steps-information exchange, deliberation, and shared decision-rooted in the ethical principles of autonomy (respect for a patient's opinions and choices), justice ("what is deserved"), and beneficence (actions to maximize benefit and minimize harm) $[9,10]$.

While many groups advocate for SDM, including the Institute of Medicine [11] and USPSTF [12], it is rarely achieved in clinical practice $[13,14]$. A nationally representative study of men considering PSA screening reported that only half were asked their preferences, and the pros and cons of screening were discussed only $32 \%$ of the time $[15,16]$. These studies suggest most prostate cancer screening decisions do not meet criteria for SDM as they lack a balanced discussion of decision consequences and preference clarification. SDM is particularly important for PSA screening among Black men, among whom the balance of risks and benefits is not definitively established but likely favor screening. Black men remain a vulnerable population and the group at highest risk of death from prostate cancer.

Decision coaching is the process by which a nonhealthcare provider coach "provides a patient with individualized, nondirective guidance to meet decisionmaking needs in preparation for consultation" with a healthcare provider [17]. Decision coaching improves incorporation of patients' values and goals into treatment decisions, promotes SDM, and improves communication between patients and providers [18-24]. Prior studies have employed nurses as decision coaches and demonstrated improvement in decision-making scores but are difficult to disseminate due to cost considerations [25, 26]. Other studies have attempted to employ lower-cost personnel as decision coaches such as college students [27]; however, in many instances, a decision coach with specific cultural sensitivities is required. A lower-cost, high-efficacy decision coach for prostate cancer screening is needed critically.

The community health worker (CHW) model is effective in communities requiring a culturally sensitive, contextualized approach to health promotion and intervention $[28,29]$. The strength of this model is derived from the multi-theoretical roots of community organizing, social support, social networks, self-efficacy, 
and peer models $[28,29]$. A community health worker is a public health worker that can provide liaison between health services and their community to facilitate access and optimize the quality and cultural competence of services delivered. A CHW intervention can be a low-cost approach to improve community health and well-being and can bridge the cultural and social barriers between underserved communities and the health care system $[11,28,30,31]$. CHWs are effective in supporting cancer screening decisions in the Black community [32, 33]. In this randomized control trial, we aim to demonstrate that CHW-led decision coaching can be a feasible, lowcost intervention to improve patient decision-making and enhance provider experience.

\section{Objectives}

Study aim

Our study proposes the following specific aims:

(1) To test whether a CHW-led decision coaching program affects decision quality, the decision-making process, patient-provider communication, and PSA utilization for Black men in the primary care setting;

(2) To assess whether a CHW-led decision coaching program improves provider experience with counseling Black men considering PSA screening;

(3) To determine the cost and budget impact of a CHW-led decision coaching program for PSA screening;

(4) To assess participant attitudes, behaviors, and norms around PSA screening and assess perceptions of the feasibility, acceptability, and sustainability of $\mathrm{CHW}$-led decision coaching in the primary care setting.

\section{Study design}

The study is a randomized control trial to evaluate the effectiveness of a CHW-led decision-coaching program to facilitate SDM for prostate cancer screening decisions among Black men at a primary care FQHC. We will enroll 228 Black men aged 40-69 years who are patients at the participating clinical site and the providers who care for them $(n=8)$. Patients will be randomized to either the intervention or the control arm of the study. Participants in the intervention arm will receive a decision aid by mail and will receive $\mathrm{CHW}$-led decision coaching on PSA screening $1 \mathrm{~h}$ before their appointment, while those in the control arm will still receive the decision aid by mail but will have a $\mathrm{CHW}$-led discussion on cardiovascular dietary and lifestyle modification rather than PSA screening; the discussion of cardiovascular risk reduction will serve as an attention control.
Patients will complete four surveys: (1) baseline at enrollment in the clinic prior to coaching, (2) immediately following coaching, but before provider consultation, (3) following provider consultation, and (4) at 6 months post-clinic visit. Providers at the FQHC will complete three surveys: (1) at study initiation, (2) after each patient encounter, and (3) at study completion immediately before participating in a semi-structured interview that will assess perceptions of the intervention's acceptability and feasibility. We will explore communication in both patient and provider groups. The protocol follows the Standard Protocol Items: Recommendations for Interventional Trials (SPIRIT) guidelines and fulfills the SPIRIT checklist (see Additional file 1). An overview of the methods is shown in Fig. 1.

\section{Sample size calculation}

In determining the recruitment target, we considered the detectable group differences in patient knowledge and decision quality based on pilot study data, differences in PSA screening rates between the control and intervention arms, and expected dropout. In our PSA pilot study, the means for knowledge change were 2.6 $(\mathrm{SD}=2.81)$ and $5.1(\mathrm{SD}=3.19)$ in the control and intervention arms, respectively. Using a two-sided Z-test for two proportions, a total group of 182 subjects with 91 subjects in each arm can achieve $80 \%$ power to detect a $20 \%$ difference in the post-intervention PSA screening rate between control and $\mathrm{CHW}$ arms at 5\% type I error. Conservatively, assuming 20\% dropout, 228 participants will be recruited to achieve a final sample of 182 (91/ arm) patients. This sample size will allow us to be sufficiently powered to detect differences in our primary study outcomes: knowledge, decision quality, and differences in PSA screening.

\section{Methods}

\section{Setting and recruitment}

This study will be performed at a primary care FQHC in New York. Data collection and decision coaching will occur at this clinic. Recruitment of eligible participants will be conducted by study staff who will review weekly electronic appointment reports and call patients using an IRB-approved recruitment script.

\section{Participant eligibility}

Consistent with prior research, we will exclude men seen within 9 months of a previous PSA test or within 180 days of a primary diagnosis of urinary obstruction, prostatitis, hematuria, disorders of prostate, unexplained weight loss, or lumbar back pain. PSA in these settings is not obtained for screening purposes [34]. Men previously diagnosed with prostate cancer (ICD-10-CM C61), 


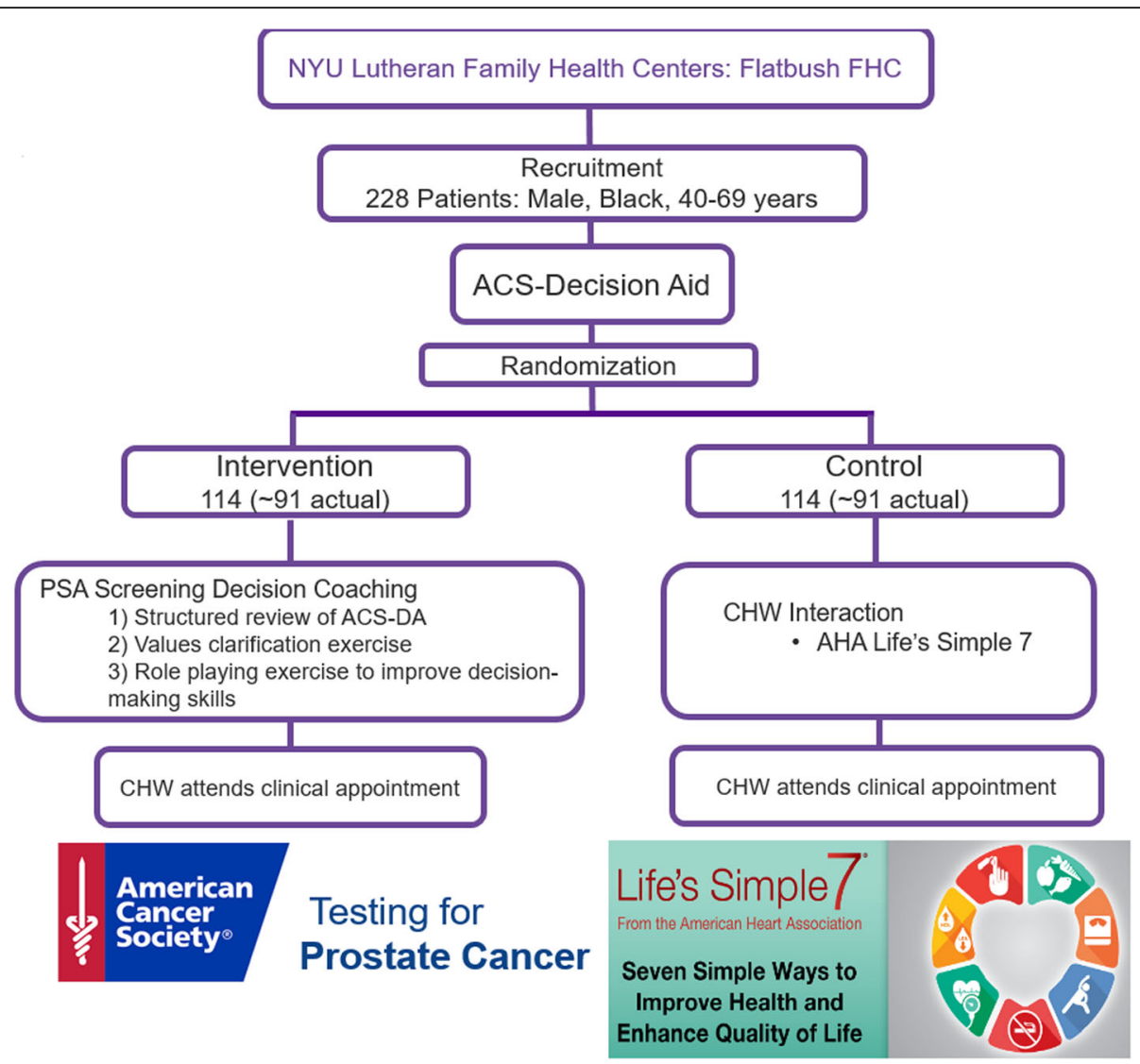

Fig. 1 Overview of the methods

and those presenting for an indication other than a well visit will be excluded.

We will enroll all eight providers who care for adult men in the FQHC: four full-time family nurse practitioners, three internal medicine physicians, and one family medicine physician. We will also enroll senior administrative staff $(n=2)$ to gain further insight on acceptability and workflow.

\section{Randomization, allocation, and blinding}

An independent randomization process will be implemented by each provider. We will stratify the randomization by age, using two strata: 40-55 years old and 55-69 years old. Within each stratum, patients (4 person blocks) will be randomized with a computergenerated permuted block random number to the control or intervention arm upon enrollment by an independent statistician. All study staff and providers who assess outcomes will be blinded to the randomization arms. Breaking of study blinding for individual participants may occur if the research coordinator finds a critical PSA result (defined as PSA $>4$ ) during the 6 months follow-up which has not been addressed by the provider.
The study team will contact the provider immediately and urge them to have a consultation with the patient.

\section{Decision aid}

This project will use the American Cancer Society Prostate Cancer Early Detection Decision Aid (ACS-DA) [6], which describes the prostate gland and its function, prostate-related problems (including cancer), prostate cancer risk factors and symptoms, prostate cancer early detection and screening paradigms, the pros and cons of PSA screening, and possible follow-up tests in response to abnormal PSA results. The ACS-DA has demonstrated effectiveness among medically underserved populations of Black men, among whom it substantially increased knowledge, lowered decisional conflict, and was well-accepted [35].

\section{CHW intervention}

In the intervention arm, the CHW coach reviews the content of the mailed decision aid and conducts a structured decision counseling session about prostate cancer screening [36]. Decision coaching will include the following components: (1) a structured discussion with the patient focusing on determining his understanding of his 
prostate cancer risk, screening options, and decisionmaking goals and values, (2) role-playing exercises to improve shared decision-making skills, and (3) coach accompaniment to the patient's appointment.

The coach will initiate the session by asking the patient about his values and goals for screening. The coach will then ask the patient what questions the patient has and what information he needs to make his decision. The coach will ascertain knowledge and ask the patient to think through the questions he would like to ask his provider. Finally, the coach will discuss any concerns the patient may have. The coach will encourage the patient to participate in role-playing exercises, allowing the patient to practice talking with his provider about treatment values, goals, and preferences.

The patient will be encouraged to make a final decision about PSA screening in consultation with his provider. At the conclusion of the coaching session, the patient and provider will receive a document summarizing the content of the interview which prioritizes questions, values, and goals. The visit will be audio-recorded to assess coaching fidelity and the quality and content of the discussion.

\section{Control arm}

Participants in the control arm will receive, but not review with a $\mathrm{CHW}$, the content of the mailed decision aid. CHW will encourage discussion of the decision aid with their provider if they inquire. The coach will offer general health coaching using an educational tool focused on dietary and lifestyle modification to reduce the risk of cardiovascular disease. Specifically, the coach will use the American Heart Association's "Life's Simple 7" educational tool [37]. Health coaching will focus on four modifiable health behaviors including smoking cessation, healthy diet, physical activity, and body mass index (BMI), and three modifiable biological factors, including blood pressure (BP), total cholesterol, and fasting glucose, as described in the control educational tool. This arm consists of the patient choosing preferred topics out of the 7 options provided. This component will serve as an attention control for the personal interaction experienced by men in the intervention arm. The coach will accompany control patients to their appointments and take notes. The visits (both intervention and control) will be audio-recorded to assess the quality, fidelity, and content of the discussion.

\section{Outcome assessment}

Outcome measurement will be based on surveys, interviews, and data collected from the medical records by trained community health workers. Primary, secondary, and exploratory outcomes are summarized in Table 1.
Table 1 Primary, secondary, and exploratory outcomes

Study endpoints/outcomes

Primary study outcomes

The following are primary study outcomes:

- Decision quality: Measure of informed choice to evaluate screening decision and attitudes towards the screening test

- Patient knowledge: Patient knowledge of prostate cancer and PSA screening

- PSA screening rates: PSA rates collected through patient selfreported PSA testing and EHR data on PSA test utilization

Secondary Study Outcomes

Secondary study outcomes are:

- Perception of quality of care: Patient perception of quality of care assessed through domains of communication, decisional self-efficacy, self-efficacy in communicating with their provider, satisfaction and decisional conflict

- Experience with decision coaching program: Provider experience with decision coaching program measured through encounter satisfaction, difficulty, and communication

Exploratory outcomes

- Net cost of CHW-led decision coaching program for PSA screening: cost of CHW-led decision coaching program and its effect on prostate cancer screening costs by measuring CHW program costs and healthcare utilization costs

- Behaviors and norms around PSA screening and perceptions of feasibility and acceptability of CHW-led decision coaching: Qualitative evaluation of patient and provider perceptions through in-depth, semi-structured interviews and other qualitative data; triangulation of mixed methods data sets from patients and provider interviews, clinical encounters, and surveys to better understand implementation.

\section{Primary outcomes Decision quality}

We will objectively measure decision quality using two domains as defined by Sepucha et al.: (1) being informed (e.g., accurate understanding about screening and its risks and benefits) and (2) making preferenceconcordant decisions (i.e., treatment consistent with patient preferences as determined by responses to survey questions) [38]. Measurement of these domains will be operationalized using the following tools and techniques:

- The Measure of Informed Choice includes 4 items assessing attitudes towards the screening test and a record of test uptake [39].

- The Decisional Balance Scale will assess the patient's attitudes towards PSA testing. The scale consists of a six-item pros scale (alpha $=0.87)$ and six-item cons scale (alpha $=0.82)$ scored on a 5-point Likert scale (strongly agree to strongly disagree) [40].

- Knowledge will be measured using a survey developed by our group that assesses understanding of prostate cancer and PSA screening [41]. The survey was piloted among Black men recruited from churches in Harlem, New York, enrolled in a study 
to help determine whether PSA screening was right for them.

- PSA screening rates will be collected 6 months postintervention through self-reported PSA testing and extracted EHR data on PSA test utilization. The association between PSA utilization and intervention exposure will then be tested.

\section{Secondary outcomes}

Patient perception of quality of care will be assessed through the domains of communication, decisional selfefficacy, self-efficacy in communication with their provider, satisfaction, and decisional conflict.

\section{- Communication:}

- The Combined Outcome Measure for Risk communication and treatment Decision-making Effectiveness (COMRADE) is a 20-item measure validated for clinical encounters [42]. Sub-scales include (1) satisfaction with physician communication and (2) patient confidence in the decision. The scale has excellent internal consistency (Cronbach's $\alpha=0.92$ ).

- The Questionnaire Concerning the DoctorPatient Communication Skills [43] is a validated 19-item scale capturing the process (greeting, listening) and content (explanations and next steps) aspects of the visit from the provider's and patient's perspectives.

- The Decision Self-Efficacy Scale is an 11-item scale measuring self-efficacy to perform informed decision-making (e.g., getting needed information, asking questions, expressing opinions, and asking for advice) [44].

- The Perceived Efficacy in Patient-Physician Interactions is a 10-item scale measuring self-efficacy for provider communication [45]. The scale is reliable (Cronbach's $\alpha=0.91$ ) and valid in older adults.

- The Satisfaction with Decision Scale is a 6-item measure assessing patient satisfaction with their decision and decision-making process [46].

- The Decisional Regret Scale is a validated, 5-item scale measuring regret or remorse following a health care decision [47].

- The Decisional Conflict Scale will measure patients' perceptions of uncertainty in choosing options, feelings of having adequate knowledge and clear values, and effective decision-making [48].

- Patient experience with the decision-coaching program will be assessed using a survey developed by our group that measures the usefulness as well as the impact of the CHW intervention on the patient and the appointment.
- Provider experience with the decision-coaching program will be measured through encounter satisfaction, difficulty, and communication.

- The Physician Satisfaction Scale designed for encounter-specific situations measures satisfaction [49]. The survey has 2 dimensions and 16 items measuring understanding of the patient's problem, perceiving patient comprehension, and affective reactions. Internal consistency is good (Cronbach $\alpha=$ $0.85)$.

- The Mental Work-Load Instrument assesses difficulty with the subjective experience or cost incurred by a physician in performing patient care [50]. The survey has 5 dimensions and 6 items addressing mental effort, physical effort, difficulty, performance, and stress. Internal consistency is good (Cronbach $\alpha=0.80)$.

- Provider communication will be assessed using the Questionnaire Concerning the Doctor-Patient Communication Skills [43].

\section{Exploratory outcomes}

Exploratory outcomes include the comparative cost of the intervention and behaviors and norms surrounding PSA screening. The cost of a CHW-led decisioncoaching program and its effect on prostate cancer screening costs will be measured by analysis of the CHW program costs and healthcare utilization costs. Intervention costs will be evaluated using the methods described by Ritzwoller et al. [51], which documents and assesses the components of the intervention and its implementation, specifies a short-term time horizon relevant to potential decision-makers who might adopt the intervention if proven effective, and outlines important sensitivity analyses for the cost analysis. We will capture CHW time associated with the intervention and apply relevant labor rates (wage plus fringe) from the Bureau of Labor Statistics. We will summarize healthcare utilization costs that emanate directly from the intervention. These include estimating costs for select screening procedures from pre-specified diagnosis and procedure codes [G0103, 84152, 84153, and 84154]. Screeningrelated activities will be ascertained beginning with the initial clinic visit and any related care as follows: costs for PSA screening; prostate biopsies performed within 180 days after a PSA test; and hospitalizations due to biopsy complications, defined as those that occurred within 30 days of prostate biopsies and have ICD-10 primary diagnosis codes consistent with complications. We will apply New York State Medicaid reimbursement rates as a lower bound estimate of healthcare costs and national average Medicare reimbursement rates as an upper bound estimate of healthcare costs. 
Behaviors and norms surrounding PSA screening will be measured using qualitative methods to identify and describe the attitudes and perceptions of Black men and their providers regarding PSA testing, the $\mathrm{CHW}$-led decision-coaching intervention, and SDM. In-depth, semi-structured interviews with both patients and providers will be conducted. The interviews will be audiorecorded and transcribed for thematic analysis.

\section{Data collection}

To evaluate the efficacy of $\mathrm{CHW}$-led decision coaching, we will collect quantitative, qualitative, and cost data.

Quantitative data will be collected from (1) surveys administered to patients and providers as well as from (2) patient clinical data collected from the institution's electronic health record, Epic Electronic Medical Record (Epic Systems Corporation).

Figure 2 shows the SPIRIT schedule of assessments and interventions for study patients. Time 1 measures are completed at enrollment in the clinic before coaching. Time 2 occurs after coaching but before provider consultation. Patients will complete Time 3 measures following their consultation. Time 4 measures are collected 6 months post-clinic visit. A subset of patients will be recruited to complete a qualitative interview at Time 4.

The SPIRIT schedule of assessments for providers is shown in Fig. 3. Providers at the FQHC will complete measures at three time points. Basic demographic information will be collected at study initiation. Measures will be collected via brief survey after each patient encounter (the provider will be blinded as to whether the patient was in the control or intervention arm). A final survey will be completed at study completion immediately before the semi-structured qualitative interview.

To promote participant retention in follow-up procedures, we will reimburse both patient and providers for their participation in the program and completing follow-up assessments.

\section{Data management}

The study case report form (CRF) is the primary data collection instrument for the study. All data will be entered into a secure, HIPAA-compliant, digital data collection system, REDCap. All data fields will be entered using free text and multiple-choice entries with reminders to ensure fields are entered appropriately. REDCap has data auditing to ensure any changes to data are recorded and justified. Hard copies will be kept in a locked storage area in a locked office and building. Access to study records will be limited to IRB-approved members of the study team.

A Data Safety and Monitoring Board (DSMB) will be established to ensure the safety of participants and the validity and integrity of the data. The DSMB will consist of a statistician (distinct from project statistician) and two pedagogical experts (distinct from study personnel). The DSMB will approve the Data and Safety Monitoring Plan and meet annually to assess overall compliance with the IRB-approved protocol. Other responsibilities of the DSMB include evaluating the progress of the study including assessments of data quality, participant risk versus benefit, and other factors that affect study outcome; making recommendations to study investigators and IRB to ensure protection of human subjects concerning continuation or conclusion of the trial; and protecting study data confidentiality.

The principal investigators are responsible for reporting adverse events to the NYU IRB, the entity granting IRB approval. Adverse events (AEs) and serious adverse events (SAEs) that are severe in nature and could potentially affect the well-being of study participants will be reported to IRB immediately. Also, the contact-PI (Dr. Makarov) will summarize AEs and SAEs in annual continuation reports.

\section{Statistical analysis}

An "intent-to-treat" (ITT) approach will be used. We will examine (1) comparability of study arms at baseline (based on chi-squared statistics or $t$-tests, as appropriate), (2) relationships between the response variables and potential covariates, and (3) predictors of missing data/dropout.

We will use linear mixed models for continuous outcomes, logistic generalized linear mixed models for binary outcomes, and random effects multinomial models for outcomes with more than 2 levels, such as adherence. In all models, time (2 dummy variables) and intervention will be included as fixed effects; provider will be a random effect. The intervention effect of interest is the treatment $\mathrm{X}$ time interaction. Identified predictors of missing data will be included as covariates in this random effect's framework, to provide unbiased estimates of the intervention effect under an assumption of missing at random (i.e., missingness depends only on observed-not on unobserved-covariates). We will conduct sensitivity analyses to assess plausible departures from this assumption. Other demographic and clinical covariates will be included as necessary in adjusted analyses. Model assessment will be conducted using appropriate regression diagnostics. The primary and secondary analyses will be done using Stata and SAS, and MPlus will be used in the mediation analyses.

Specifically, to determine the effect of the intervention on the primary outcomes, a random effects (generalized) linear regression model will be used to test absolute and time-specific differences attributable to the intervention. In additional analyses, we will adjust for other covariates 


\begin{tabular}{|c|c|c|c|c|c|c|}
\hline \multirow[t]{2}{*}{ Quantitative Patient Measures } & \multicolumn{6}{|c|}{ Collection Time } \\
\hline & $\begin{array}{l}\text { Before } \\
\text { enrollment } \\
\left(\begin{array}{lll}-7 & \text { to } & -28 \\
\text { days })\end{array}\right.\end{array}$ & $\begin{array}{l}\text { Time } \\
1\end{array}$ & Intervention & $\begin{array}{l}\text { Time } \\
2\end{array}$ & $\begin{array}{l}\text { Time } \\
3\end{array}$ & $\begin{array}{l}\text { Time } \\
4\end{array}$ \\
\hline Recruitment & $\mathrm{X}$ & & & & & \\
\hline Eligibility screen & $\mathrm{X}$ & & & & & \\
\hline Informed consent & & $\mathrm{X}$ & & & & \\
\hline Enrollment & & $\mathrm{X}$ & & & & \\
\hline Randomization & & $\mathrm{X}$ & & & & \\
\hline Intervention & & & $\mathrm{X}$ & & & \\
\hline \multicolumn{7}{|l|}{ ASSESSMENTS } \\
\hline \multicolumn{7}{|l|}{ Demographics, Clinical Features and Preferences } \\
\hline Demographics & & $\mathrm{X}$ & & & & \\
\hline Literacy $y^{45}$ and Numeracy $y^{46,47}$ & & $\mathrm{X}$ & & & & \\
\hline Trust $^{60,61}$ & & $\mathrm{X}$ & & & & \\
\hline International Prostate Symptom Score & & $\mathrm{X}$ & & & & \\
\hline Health Survey & & $\mathrm{X}$ & & & & \\
\hline Preference for Shared Decision Making ${ }^{62}$ & & $\mathrm{X}$ & & $\mathrm{X}$ & $\mathrm{X}$ & \\
\hline \multicolumn{7}{|l|}{ Primary Outcomes } \\
\hline Knowledge $^{42}$ & & & & $\mathrm{X}$ & $\mathrm{X}$ & \\
\hline Measure of Informed Choice ${ }^{40}$ & & & & $\mathrm{X}$ & $\mathrm{X}$ & \\
\hline Decision Quality ${ }^{39,41}$ & & & & $\mathrm{X}$ & $\mathrm{X}$ & \\
\hline Screening choice (Epic Query) & & & & & $\mathrm{X}$ & $\mathrm{X}$ \\
\hline \multicolumn{7}{|l|}{ Secondary Outcomes } \\
\hline Communication $^{43,44}$ & & & & & $\mathrm{X}$ & \\
\hline Decision Self-Efficacy Scale ${ }^{50}$ & & $\mathrm{X}$ & & $\mathrm{X}$ & $\mathrm{X}$ & \\
\hline Self-efficacy for communicating with provider ${ }^{51}$ & & $\mathrm{X}$ & & $\mathrm{X}$ & $\mathrm{X}$ & \\
\hline Decisional conflict scale ${ }^{54}$ & & & & $\mathrm{X}$ & $\mathrm{X}$ & $\mathrm{X}$ \\
\hline Satisfaction with Decision Scale $^{52}$ & & & & & $\mathrm{X}$ & $\mathrm{X}$ \\
\hline Decisional Regret ${ }^{53}$ & & & & & & $\mathrm{X}$ \\
\hline Experience with decision coaching program & & & & & $\mathrm{X}$ & \\
\hline
\end{tabular}

Fig. 2 Quantitative patient measures

which may be unbalanced between the intervention arms at baseline at $p=0.10$. For hypotheses pertaining to patients' perception of care quality, we will model satisfaction with provider communication, self-efficacy, difficulty, and decisional conflict in separate analyses of control and intervention arms to examine possible associations between those measurements and the outcomes.
We will summarize costs using descriptive statistics, including mean, medians, and standard deviations, for each intervention component including downstream costs outlined above. We will tally all costs to estimate the intervention's budget impact and the total average cost per patient. We will conduct extensive sensitivity analyses, including a range of relevant salaries for 


\begin{tabular}{|c|c|c|c|}
\hline \multicolumn{4}{|c|}{ Quantitative Provider Measures } \\
\hline Measure & Initiation & Post encounter & Completion \\
\hline \multicolumn{4}{|c|}{$\begin{array}{l}\text { Demographics and } \\
\text { Preferences }\end{array}$} \\
\hline Demographics & $\mathrm{X}$ & & \\
\hline Preference for $\mathrm{EBM}^{48}$ & $\mathrm{X}$ & & $\mathrm{X}$ \\
\hline \multicolumn{4}{|l|}{ Specific Aim 2} \\
\hline \multicolumn{4}{|l|}{ Hypothesis 2.1} \\
\hline Satisfaction $^{55}$ & & $\mathrm{X}$ & \\
\hline Difficulty $^{56}$ & & $\mathrm{X}$ & \\
\hline Communication $^{44}$ & & $\mathrm{X}$ & \\
\hline
\end{tabular}

Fig. 3 Quantitative provider measures

providers and staff and upper and lower bounds for estimating the cost of healthcare utilization.

\section{Limitations}

It may not be possible to blind providers completely, especially with the CHW Decision Coach's presence at the clinic visit. The discussion between coach and patient may reveal their previous interaction. Providers may thus be prompted to engage in greater SDM than they might have outside of the study. Providers will also be handed a document with a prompt to identify the patient as part of the study and to remind the provider to engage in a discussion of prostate cancer screening during the visit. This is likely to spur SDM type discussions in both control and interventions arms, biasing our results towards the null and making a type I error less likely.

\section{Discussion}

In this trial, we evaluate the efficacy of a CHW-led decision-coaching program to facilitate shared decisionmaking for PSA screening among Black men at a primary care FQHC. CHW-led interventions are known to improve awareness, knowledge, support, and efficacy to reduce the impact of chronic disease and cancer in underserved populations [52-56]. Thus, CHWs seem ideally suited as decision coaches in primary care practices seeking to facilitate SDM for PSA screening among
Black men, which has not been tested previously. A rigorous study design will be applied to this question with an independent randomization process and blinding of all individuals who assess study outcomes, including healthcare providers, the statistician, to reduce bias.

\section{Trial status}

This trial is ongoing. Recruitment began on June 19, 2019, and will continue until September 2021. The trial procedures are expected to be completed by the end of March 2023.

Protocol version 02.26.20.

\section{Supplementary Information}

The online version contains supplementary material available at https://doi. org/10.1186/s13063-021-05064-4.

\section{Additional file 1.}

\section{Abbreviations}

CFR: Code of Federal Regulations; CHW: Community health worker; CRF: Case report form; DSMB: Data and Safety Monitoring Board; FQHC: Federally Qualified Health Center; HIPAA: Health Insurance Portability and Accountability Act; IRB: Institutional Review Board; N: Number (typically refers to participants); NIH: National Institutes of Health; PSA: Prostate-specific antigen; SDM: Shared decision-making; US: United States

\section{Acknowledgements}

We gratefully acknowledge the contributions of Jerry Thomas in preparing this article. The authors are grateful to the research assistants, coordinators, and community health workers at all participating sites. 


\section{Authors' contributions}

$\mathrm{DM}$ and JR co-led the conception of the trials with contributions from all other authors. DM and JR led on trial design; DM, ZF, SC, NML, and JR drafted the manuscript with contributions from MS on qualitative methods and CHW-led interventions; HTG on cost analyses; HL on statistical methodology; AF on risk communication, development of decision aids, decision support interventions, and decision coach training; PU on patient-physician encounter analysis to understand SDM; and RW on ethical and implementation guidance. The authors read and approved the final manuscript.

\section{Funding}

This study is financed through a grant by the National Institute On Minority Health and Health Disparities (NIMHD) of the National Institutes of Health under Award Number R01MD012243.

Any sponsor-related questions for the study may be directed to the NIMHD Program Director Rick Berzon, DrPH, PA at Rick.Berzon@nih.gov or 301-5948949. The sponsor played no part in study design; collection, management, analysis, and interpretation of data; writing of the report; and the decision to submit the report for publication.

\section{Availability of data and materials}

This study will comply with the NIH Public Access Policy, which ensures that the public has access to the published results of $\mathrm{NIH}$ funded research. It requires scientists to submit final peer-reviewed journal manuscripts that arise from NIH funds to the digital archive PubMed Central upon acceptance for publication.

\section{Ethics approval and consent to participate}

This study will be conducted in full conformity with Regulations for the Protection of Human Subjects of Research codified in 45 CFR Part 46. The protocol, informed consent form(s), recruitment materials, and all participant materials have been reviewed and approved by the New York University School of Medicine Institutional Review Board (i18-00514). Any amendment to the protocol will require review and approval by the IRB before the changes are implemented to the study. All changes to the consent form will be IRB approved; a determination will be made regarding whether previously consented participants need to be re-consented.

Consent forms describing in detail the study intervention, study procedures, and risks are given to the participant and written documentation of informed consent is required prior to starting intervention. All participants will receive a verbal explanation in terms suited to their comprehension of the purposes, procedures, and potential risks of the study and of their rights as research participants. Participants will have the opportunity to carefully review the written consent form and ask questions prior to signing. The participants should have the opportunity to discuss the study with their surrogates or think about it prior to agreeing to participate. The participant will sign the informed consent document prior to any procedures being done specifically for the study. Subjects will sign written full informed consent and audio consent form prior to enrollment in the study.

\section{Consent for publication}

Not applicable.

\section{Competing interests}

The authors have no financial or non-financial competing interests to be declared.

\section{Author details}

'VA New York Harbor Healthcare System, 423 E 23rd St, New York, NY, USA. ${ }^{2}$ Departments of Urology, NYU Langone Health, 227 E 30th St, New York, NY, USA. ${ }^{3}$ Population Health, NYU Langone Health, 227 E 30th St, New York, NY, USA. ${ }^{4}$ Department of Population Health Sciences, University of Utah School of Medicine, Salt Lake City, UT, USA. ${ }^{5}$ NYU College of Nursing, 433 First Avenue, New York, NY, USA. ${ }^{6}$ Sunset Park Health Council, Brooklyn, NY, USA. ${ }^{7}$ National Center for Bioethics in Research and Health Care, Tuskegee University, Tuskegee, USA. ${ }^{8}$ The Fuqua School of Business, Duke University, Durham, NC, USA.
Received: 21 September 2020 Accepted: 20 January 2021 Published online: 10 February 2021

\section{References}

1. Cronin KA, Lake AJ, Scott S, Sherman RL, Noone AM, Howlader N, et al. Annual report to the nation on the status of cancer, part I: national cancer statistics. Cancer. 2018;124(13):2785-800.

2. American Cancer Society. Cancer Facts \& Figures for African Americans 2019-2021. Am Cancer Soc 2019. Available from: https:/www.cancer.org/ content/dam/cancer-org/research/cancer-facts-and-statistics/cancer-factsand-figures-for-african-americans/cancer-facts-and-figures-for-africanamericans-2019-2021.pdf. Accessed 18 Sept 2019.

3. Du XL, Fang S, Coker AL, Sanderson M, Aragaki C, Cormier JN, et al. Racial disparity and socioeconomic status in association with survival in older men with local/regional stage prostate carcinoma. Cancer. 2006;106(6):1276-85.

4. Sandblom G, Varenhorst E, Rosell J, Löfman O, Carlsson P. Randomised prostate cancer screening trial: 20 year follow-up. BMJ. 2011;342:d1539.

5. Kjellman A, Akre O, Norming U, Törnblom M, Gustafsson O. 15-year followup of a population based prostate cancer screening study. J Urol. 2009;181(4):1615-21.

6. Testing for Prostate Cancer. In: American Cancer Society; 2015. https://www. cancer.org/content/dam/cancer-org/cancer-control/en/booklets-flyers/ testing-forprostate-cancer-handout.pdf. Accessed 30 Sept 2019.

7. Taylor KL, Davis JL, Turner RO, Johnson L, Schwartz MD, Kerner JF, et al. Educating African American men about the prostate cancer screening dilemma: a randomized intervention. Cancer Epidemiol Biomark Prev. 2006; 15(11):2179-88.

8. American Urological Association. Early Detection of Prostate Cancer (2018) [Internet]. American Urological Association. 2018. Available from https:// www.auanet.org/guidelines/prostate-cancer-early-detection-guideline. Accessed 30 Sept 2019.

9. National Commision for the Protection of Human Subjects of Biomedical and Behavioral Research. The Belmont Report: Ethical Principles and Guidelines for the Protection of Human Subjects of Research [Internet]. Bethesda: The Commission; 1979 [cited 2019 Sep 18]. Available from https:/ www.hhs.gov/ohrp/regulations-and-policy/belmont-report/read-thebelmont-report/index.html.

10. Charles C, Gafni A, Whelan T. Shared decision-making in the medical encounter: what does it mean? (or it takes at least two to tango). Soc Sci Med. 1997;44(5):681-92.

11. Institute of Medicine (US) Committee on Quality of Health Care in America. Crossing the quality chasm: a new health system for the 21st century. Washington DC: National Academies Press (US); 2001. Available from https://pubmed.ncbi.nlm.nih.gov/25057539/. Accessed 30 Sept 2019

12. Published Final Recommendation Summary: prostate cancer: screening. US Preventive Services Task Force 2018. https://www. uspreventiveservicestaskforce.org/uspstf/recommendation/prostate-cancerscreening. Accessed 30 Sep 2019.

13. Makarov DV, Chrouser K, Gore JL, Maranchie J, Nielsen M, et al. AUA white paper on implementation of shared decision making into urological practice. Urology Practice. 2016;3(5):355-63.

14. Legare F, Elwyn G, Fishbein M, Fremont P, Frosch D, Gagnon MP, et al. Translating shared decision-making into health care clinical practices: proof of concepts. Implement Sci. 2008;3:2. https://doi.org/10.1186/1748-5908-3-2.

15. Hoffman RM, Lewis CL, Pignone MP, Couper MP, Barry MJ, Elmore JG, et al. Decision-making processes for breast, colorectal, and prostate cancer screening: the DECISIONS survey. Med Decis Mak. 2010;30(5 Suppl):53S-64S.

16. Hoffman RM, Couper MP, Zikmund-Fisher BJ, et al. Prostate cancer screening decisions: results from the National Survey of Medical decisions (DECISIONS study). Arch Intern Med. 2009;169(17):1611-8.

17. Stacey D, Kryworuchko J, Bennett C, Murray MA, Mullan S, Légaré F. Decision coaching to prepare patients for making health decisions a systematic review of decision coaching in trials of patient decision aids. Med Decis Mak. 2012;32(3):E22-33.

18. Sepucha KR, Belkora JK, Tripathy D, Esserman L. Building bridges between physicians and patients: results of a pilot study examining new tools for collaborative decision making in breast cancer. J Clin Oncol. 2000;18(6): 1230-8.

19. Haywood K, Marshall S, Fitzpatrick R. Patient participation in the consultation process: a structured review of intervention strategies. Patient Educ Couns. 2006;63(1-2):12-23. 
20. Cegala DJ. Emerging trends and future directions in patient communication skills training. Health Commun. 2006;20(2):123-9.

21. Cegala DJ, Post DM. On addressing racial and ethnic health disparities: the potential role of patient communication skills interventions. Am Behav Sci. 2006;49(6):853-67.

22. Street RL Jr, Slee C, Kalauokalani DK, Dean DE, Tancredi DJ, Kravitz RL Improving physician-patient communication about cancer pain with a tailored education-coaching intervention. Patient Educ Couns. 2010;80(1): 42-7.

23. Davison BJ, Degner LF. Empowerment of men newly diagnosed with prostate cancer. Cancer Nurs. 1997;20(3):187-96.

24. Griffin SJ, Kinmonth AL, Veltman MW, Gillard S, Grant J, Stewart M. Effect on health-related outcomes of interventions to alter the interaction between patients and practitioners: a systematic review of trials. Ann Fam Med. 2004; 2(6):595-608.

25. Myers RE, Daskalakis C, Kunkel EJ, Cocroft JR, Riggio JM, Capkin M, et al. Mediated decision support in prostate cancer screening: a randomized controlled trial of decision counseling. Patient Educ Couns. 2011;83(2):2406.

26. Myers RE, Daskalakis C, Cocroft J, Kunkel EJ, Delmoor E, Liberatore M, et al. Preparing African-American men in community primary care practices to decide whether or not to have prostate cancer screening. J Natl Med Assoc. 2005;97(8):1143-54.

27. Belkora J, Volz S, Loth M, Teng A, Zarin-Pass M, Moore D, et al. Coaching patients in the use of decision and communication aids: RE-AIM evaluation of a patient support program. BMC Health Serv Res. 2015;15:209.

28. Love MB, Gardner K, Legion V. Community health workers: who they are and what they do. Health Educ Behav. 1997;24(4):510-22.

29. Wilson K, Brownstein J, Blanton C. Community health advisor use: insights from a national survey. In: Centers for Disease Control and Prevention. Community health advisors/community health workers: selected annotations and programs in the United States, Vol. III. Atlanta: Centers for Disease Control and Prevention, National Center for Chronic Disease Prevention and Health Promotion; 1998. p. 11-9.

30. Pew Health Professions Commission. Community health workers: integral yet often overlooked members of the health care workforce. San Francisco: University of California at San Francisco Center for Health Professions; 1994

31. Brownstein JN, Bone LR, Dennison CR, Hill MN, Kim MT, Levine DM. Community health workers as interventionists in the prevention and control of heart disease and stroke. Am J Prev Med. 2005;29(5):128-33.

32. Boyd AS, Wilmoth MC. An innovative community-based intervention for African American women with breast cCancer: the witness project. Health Soc Work. 2006;31(1):77-80.

33. Shelton RC, Dunston SK, Leoce N, Jandorf L, Thompson HS, Erwin DO. Advancing understanding of the characteristics and capacity of African American women who serve as lay health advisors in community-based settings. Health Educ Behav. 2017:44(1):153-64.

34. Walter LC, Bertenthal D, Lindquist K, Konety BR. PSA screening among elderly men with limited life expectancies. JAMA. 2006;296(19):2336-42.

35. Gökce Ml, Wang X, Frost J, Roberson P, Volk R, Brooks D, et al. Informed decision making before prostate-specific antigen screening: initial results using the American Cancer Society (ACS) decision aid (DA) among medically underserved men. Cancer. 2017;123(4):583-91.

36. Myers RE. Decision counseling in cancer prevention and control. Health Psychol. 2005;24(4S):S71-7.

37. American Heart Association. Life's Simple 7. 2020. https://playbook.heart.org/ lifes-simple-7. Accessed 25 Feb 2020.

38. Sepucha K, Ozanne E, Silvia K, Partridge A, Mulley AG Jr. An approach to measuring the quality of breast cancer decisions. Patient Educ Couns. 2007; 65(2):261-9.

39. Marteau TM, Dormandy E, Michie S. A measure of informed choice. Health Expect. 2001;4(2):99-108.

40. Watson E, Hewitson P, Brett J, Bukach C, Evans E, Edwards A, et al. Informed decision making and prostate specific antigen (PSA) testing for prostate cancer: a randomised controlled trial exploring the impact of a brief patient decision aid on men's knowledge, attitudes and intention to be tested. Patient Educ Couns. 2006;63(3):367-79.

41. Fenstermaker M, Hickman T, Gold H, Makarov D, Loeb S, Cole H, et al. Abstract A15: how does doctor-patient communication about prostate cancer screening influence African-American patients' decisional conflict around screening decisions? Cancer Epidemiol Biomark Prev. 2016; https:// doi.org/10.1158/1538-7755.DISP15-A15.

42. Edwards A, Elwyn G, Hood K, Robling M, Atwell C, Holmes-Rovner M, et al. The development of COMRADE--a patient-based outcome measure to evaluate the effectiveness of risk communication and treatment decision making in consultations. Patient Educ Couns. 2003;50(3):311-22.

43. Campbell C, Lockyer J, Laidlaw T, Macleod H. Assessment of a matched-pair instrument to examine doctor-patient communication skills in practising doctors. Med Educ. 2007:41(2):123-9.

44. O'Connor AM. User Manual - Decision Self-Efficacy Scale. 1995. https:// decisionaid.ohri.ca/docs/develop/User_Manuals/UM_Decision_SelfEfficacy. pdf. Accessed 18 Sep 2019.

45. Maly RC, Frank JC, Marshall GN, DiMatteo MR, Reuben DB. Perceived efficacy in patient-physician interactions (PEPPI): validation of an instrument in older persons. J Am Geriatr Soc. 1998;46(7):889-94.

46. Holmes-Rovner M, Kroll J, Schmitt N, Rovner DR, Breer ML, Rothert ML, et al. Patient satisfaction with health care decisions: the satisfaction with decision scale. Med Decis Mak. 1996;16(1):58-64.

47. Brehaut JC, O'Connor AM, Wood TJ, Hack TF, Siminoff L, Gordon E, et al. Validation of a decision regret scale. Med Decis Mak. 2003;23(4):281-92.

48. O'Connor AM. Validation of a decisional conflict scale. Med Decis Mak. 1995; 15(1):25-30.

49. Shore BE, Franks P. Physician satisfaction with patient encounters: reliability and validity of an encounter-specific questionnaire. Med Car. 1986;24(7): 580-9.

50. Bertram DA, Opila DA, Brown JL, Gallagher SJ, Schifeling RW, Snow IS, et al. Measuring physician mental workload: reliability and validity assessment of a brief instrument. Med Care. 1992;30(2):95-104.

51. Ritzwoller DP, Sukhanova A, Gaglio B, Glasgow RE. Costing behavioral interventions: a practical guide to enhance translation. Ann Behav Med. 2009:37(2):218-27.

52. Gary TL, Batts-Turner M, Bone LR, Yeh HC, Wang NY, Hill-Briggs F, et al. A randomized controlled trial of the effects of nurse case manager and community health worker team interventions in urban African-Americans with type 2 diabetes. Control Clin Trials. 2004;25(1):53-66.

53. Vetter MJ, Bristow L, Ahrens J. A model for home care clinician and home health aide collaboration: diabetes care by nurse case managers and community health workers. Home Healthc Nurse. 2004;22(9):645-8.

54. Staten LK, Gregory-Mercado KY, Ranger-Moore J, Will JC, Giuliano AR, Ford ES, et al. Provider counseling, health education, and community health workers: the Arizona WISEWOMAN project. J Women's Health. 2004;13(5): 547-56.

55. Aubert RE, Herman WH, Waters J, Moore W, Sutton D, Peterson BL, et al. Nurse case management to improve glycemic control in diabetic patients in a health maintenance organization. A randomized, controlled trial. Ann Intern Med. 1998;129(8):605-12.

56. Kim K, Choi JS, Choi E, Nieman CL, Joo JH, Lin FR, et al. Effects of community-based health worker interventions to improve chronic disease management and care among vulnerable populations: a systematic review. Am J Public Health. 2016;106(4):e3-e28.

\section{Publisher's Note}

Springer Nature remains neutral with regard to jurisdictional claims in published maps and institutional affiliations.

\section{Ready to submit your research? Choose BMC and benefit from:}

- fast, convenient online submission

- thorough peer review by experienced researchers in your field

- rapid publication on acceptance

- support for research data, including large and complex data types

- gold Open Access which fosters wider collaboration and increased citations

- maximum visibility for your research: over $100 \mathrm{M}$ website views per year

At BMC, research is always in progress.

Learn more biomedcentral.com/submissions 